\title{
RELACIÓN ENTRE AUTOESTIMA Y ESTRATEGIAS DE REGULACIÓN EMOCIONAL EN ESTUDIANTES CON ALTA CAPACIDAD QUE PARTICIPAN DE UN PROGRAMA DE ENRIQUECIMIENTO EXTRACURRICULAR CHILENO ${ }^{1}$
}

\author{
Gracia Navarro Saldaña ${ }^{2}$ \\ Gabriela Flores-Oyarzo ${ }^{3}$ \\ Josefina Rivera Illanes ${ }^{4}$
}

RESUMEN

La autoestima global y la regulación emocional contribuyen al bienestar, la adaptación social y el desarrollo integral de las personas, ambas escasamente estudiadas en estudiantes con alta capacidad (AC), principalmente en el contexto chileno y latinoamericano, de ahí su importancia. En este estudio se busca identificar el nivel de autoestima global, reconocer la estrategia de regulación emocional utilizada y la relación entre autoestima global y estrategias de regulación emocional en 37 estudiantes con alta capacidad que participan de un programa de enriquecimiento extracurricular chileno. Para ello, se utiliza la Escala de Autoconcepto para niños y jóvenes de Piers-Harris, en la versión de Gorostegui, y el Cuestionario de Regulación Emocional para niños y adolescentes (ERQ-CA), versión revisada por Gullone y Taffe (2012). Se realiza análisis descriptivo y de correlación de variables, dando cuenta de que estos estudiantes poseen adecuados niveles de autoestima global, utilizan mayoritariamente la reevaluación cognitiva como estrategia de regulación emocional y existe una correlación negativa, estadísticamente significativa, entre la autoestima global y la supresión expresiva. Este estudio se instala como un aporte en el conocimiento de cualidades socioafectivas de estudiantes con AC y releva la importancia de generar espacios educativos orientados a la satisfacción integral y sistemática de sus necesidades.

Conceptos clave: autoestima, estudiante excepcional, emoción, inhibición, cognición, educación emocional, inteligencia emocional, inteligencias múltiples, competencias básicas, conciencia emocional, regulación emocional

1 En este estudio se utiliza los artículos "el" y "los" para hacer referencia tanto a hombres como a mujeres, ya sea en singular o plural, según corresponda.

2 Universidad de Concepción, Concepción, Chile. Contacto: gnavarro@udec.cl

3 Universidad de Concepción, Concepción, Chile. Contacto: gabflores@udec.cl

4 Universidad de Concepción, Concepción, Chile. Contacto: josefrivera@udec.cl 


\title{
RELATIONSHIP BETWEEN SELF ESTEEM AND EMOTIONAL REGULATION STRATEGIES AMONG GIFTED STUDENTS PARTICIPATING IN A CHILEAN EXTRACURRICULAR ENRICHMENT PROGRAM
}

\begin{abstract}
Global self-esteem and Emotional Regulation contribute to the well-being, social adaptation and comprehensive development of people, both of which are scarcely studied in Gifted students, especifically in the Chilean and Latin American context, hence their importance. This study seeks to identify the level of global self-esteem, recognize the emotional regulation strategy used and the relationship between global self-esteem and emotional regulation strategies in 37 Gifted students participants in a Chilean extracurricular enrichment program. To this effect, the Piers-Harris Self-Concept Scale for children and young people, in Gorostegui's version, and the Emotional Regulation Questionnaire for children and adolescents (ERQ-CA), version revised by Gullone and Taffe (2012) are used. A descriptive analysis and a correlation analysis of variables are carried out, revealing that these students have adequate levels of global self-esteem, mostly use cognitive reassessment as an emotional regulation strategy, and there is a statistically significant negative correlation between global self-esteem and expressive suppression. This study becomes a contribution to the knowledge of socio-affective qualities of Gifted students and highlights the relevance of creating educational spaces focused on the satisfaction of their needs in a comprehensive and systematic manner.
\end{abstract}

Key concepts: self-esteem, gifted students, emotion, inhibition, cognition, emotional education, social and emotional learning, emotional intelligence, multiple intelligences, key competencies, emotional conscience 
8 RELACIÓN ENTRE AUTOESTIMA Y ESTRATEGIAS DE REGULACIÓN EMOCIONAL EN ESTUDIANTES CON ALTA CAPACIDAD QUE PARTICIPAN DE UN PROGRAMA DE ENRIQUECIMIENTO EXTRACURRICULAR CHILENO - G. Navarro Saldaña, G. Flores-Oyarzo y J. Rivera Illanes

\section{Introducción}

El presente estudio buscó comprender la relación entre el nivel de autoestima y las estrategias de regulación emocional utilizadas por estudiantes con alta capacidad, entre 10 y 12 años, que participan de un programa de enriquecimiento extracurricular (Talentos UdeC).

Los participantes de este estudio fueron estudiantes con alta capacidad (AC), con alto potencial intelectual expresado de manera espontánea. Contaban con cualidades socioafectivas que los diferenciaban de sus pares sin este potencial, y necesidades educativas especiales asociadas a su potencial intelectual (Gagné, 2015; Miguel y Moya, 2011). Tenían entre 10 y 12 años de edad, por tanto, se encontraban en la etapa del desarrollo denominada "infancia tardía", caracterizada por una serie de cambios cognitivos, físicos y socioafectivos que afectaban su identidad, emociones y relaciones, entre otros aspectos (Delgado, 2009; Di Blas \& Cepollaro, 2017; López, 2018; Mansilla, 2000; Papalia, Feldman y Martorell, 2012; Quispe, 2019; Sabatier, Restrepo, Moreno, Hoyos \& Palacio, 2017), y participaban de un programa de enriquecimiento extracurricular que cuenta con un modelo educativo cuyo propósito es brindar una oportunidad educativa disciplinar y socioafectiva para la satisfacción de las necesidades educativas especiales de estudiantes con AC (Pirovano, 2019; Sastre-Riba, 2014; Pérez, Furlan, Heredia, y Lescano, 2015; Valadez y Ávalos, 2010).

El estudio de la autoestima global y las estrategias de regulación emocional en este tipo de estudiantes, si bien es abundante en el mundo, es escaso en contexto chileno, de ahí la importancia de su estudio. La "autoestima global" se entiende como aquella autoevaluación y valoración, positiva o negativa, que hace una persona respecto de sus cualidades, habilidades y características percibidas (Marmolejo y Romero, 2016; Mejía, 2007; Montoya y Sol, 2001; Vicent, 2018), y las "estrategias de regulación emocional" como aquellos mecanismos por medio de los cuales se modifican o modulan las emociones (Gómez y Calleja, 2016). 
En consideración a las cualidades y necesidades de estudiantes con AC, que tienen entre 10 y 12 años y participan de un programa de enriquecimiento, parece relevante, por una parte, conocer el nivel de autoestima global y las estrategias de regulación emocional que utilizan estos estudiantes, y, por otra, comprender la relación entre el nivel de autoestima global con el tipo de estrategia de regulación emocional utilizada. Esto permitiría dar luces acerca de algunas necesidades socioafectivas de estos estudiantes, y aportaría a la generación de conocimiento para la generación de oportunidades educativas oportunas y eficaces.

\section{Infancia tardía}

La infancia tardía comprende el periodo entre los 9 y 12 años, aproximadamente. Es una etapa de profundo conocimiento y experiencias sociales nuevas, en la cual el infante desarrolla de manera gradual la capacidad de compararse con otros, considerar la retroalimentación de sus seres queridos y figuras significativas en la evaluación de sí mismo, y construir su propio yo en la medida en que integra los aprendizajes adquiridos a lo largo de su vida y moldea una personalidad que le diferencia ante los demás (Di Blas \& Cepollaro, 2017; López, 2018; Quispe, 2019).

En términos psicosociales, en esta etapa del ciclo vital el autoconcepto se torna más complejo, pues ellos perciben sus habilidades y competencias en diversos ámbitos, entre los cuales está el aspecto social, físico, académico y familiar, lo cual afecta su autoestima, los pares se vuelven más influyentes y son de gran relevancia, generando relaciones de amistad más sólidas e íntimas, son capaces de mostrar comportamientos y respuestas de regulación emocional más ajustadas socialmente, disminuye el egocentrismo, tienen mayor conciencia del efecto de sus emociones sobre otros y el entorno, y aparecen las estrategias cognitivas para la regulación de las emociones (Delgado, 2009; Mansilla, 2000; Papalia et al., 2012; Quispe, 2019; Sabatier et al., 2017). 
10 RELACIÓN ENTRE AUTOESTIMA Y ESTRATEGIAS DE REGULACIÓN EMOCIONAL EN ESTUDIANTES CON ALTA CAPACIDAD QUE PARTICIPAN DE UN PROGRAMA DE ENRIQUECIMIENTO EXTRACURRICULAR CHILENO - G. Navarro Saldaña, G. Flores-Oyarzo y J. Rivera Illanes

\section{Alta capacidad}

Ahora bien, los participantes de este estudio no solo contaban con características propias de la etapa del ciclo vital en que se encontraban, sino también las que corresponden a la alta capacidad.

La alta capacidad (AC) puede comprenderse como un símil al componente "dotación intelectual" del modelo de Gagné (2015), constituyéndose como la presencia de altas aptitudes o dones intelectuales que no han sido entrenados, y que se manifiestan de manera espontánea en el área intelectual, los cuales, en tanto potencial, implican la latencia de aptitudes para el desarrollo del talento académico (Gagné, 2015; Miguel y Moya, 2011). Pero para transformar este potencial intelectual en talento académico, se requiere, por una parte, de un entorno estimulante, que brinde oportunidades sistemáticas, flexibles y focalizadas para su máximo y óptimo desarrollo, poniendo a las aptitudes naturales en interacción con catalizadores contextuales e interpersonales. Por otro lado, se requiere motivación personal, expresada en el proceso de aprendizaje, y energía y disposición para enfrentar las posibles dificultades (Acereda y Sastre, 1998; Mendioroz, Rivero y Aguilera, 2019; Pérez et al., 2015; Rodríguez, 2013; Valadez y Ávalos, 2010; Worrell, Subotnik, Olszewski-Kubilius \& Dixson, 2019).

En este estudio se entenderá al AC como la presencia de capacidades o aptitudes intelectuales que se expresan de manera espontánea y sin entrenamiento previo, constituyéndose como un potencial intelectual superior al 90\% de la población del mismo rango etario y que, en tanto potencial, requiere que la persona se comprometa en el aprendizaje, entrenamiento y práctica sistemática para transformarlo en desempeño sobresaliente o talento académico. Esta AC dota al estudiante de potencial para tener un desempeño sobresaliente en actividades académicas, el cual se expresará en la medida en que sus necesidades educativas y socioafectivas sean atendidas, y esté en interacción con catalizadores ambientales que movilicen su motivación, esfuerzo y perseverancia. Así, para este estudio, la AC es sinónimo de "potencial de talento académico", pero no es lo mismo que el talento académico. 
En este sentido, los programas de enriquecimiento extracurricular surgen como una respuesta para satisfacer las necesidades educativas especiales (NEE) de estudiantes con AC, brindando una oportunidad educativa que les permite profundizar en aprendizajes tanto disciplinares como socioafectivos, centrándose en el reconocimiento no solo de las necesidades de estos estudiantes, sino también en sus capacidades e intereses (Pirovano, 2019; SastreRiba, 2014; Pérez et al., 2015; Valadez y Ávalos, 2010).

En Chile, luego de que algunos estudios realizados en la década de los noventa dieran cuenta de la presencia de estudiantes con alto potencial intelectual en el sistema escolar regular, se comienzan a implementar programas de enriquecimiento para niños, niñas y jóvenes con potencial de talento académico, partiendo en Santiago, en 2001, con el programa PENTA UC, de la Pontificia Universidad Católica de Chile (Conejeros, Cáceres y Riveros, 2012).

A la fecha, además del PENTA UC, hay seis programas de enriquecimiento para estudiantes con potencial de talento académico en funcionamiento: DELTA UCN, de la Universidad Católica del Norte en Antofagasta, desde 2004; PROENTA UFRO, de la Universidad de la Frontera en Temuco, desde 2004; TALENTOS UdeC, de la Universidad de Concepción, en Concepción, desde 2004; BETA PUCV, de la Pontifica Universidad Católica de Valparaíso, en Valparaíso, desde 2005, ALTA UACh, de la Universidad Austral de Chile, en Valdivia, desde 2009 y Semilla UCM, de la Universidad Católica del Maule, en Talca, desde 2016. Estos programas buscan, entre algunos aspectos, ofrecer oportunidades educativas de alta calidad, que satisfagan las necesidades educativas especiales de niños, niñas y jóvenes con alta dotación intelectual, en las que, de acuerdo con sus áreas de interés, puedan enriquecer y profundizar las experiencias que ofrece el sistema escolar regular, permitiendo a sus participantes la adaptación socioemocional y adquirir conocimientos y habilidades que potencien sus talentos, y comprometerse voluntariamente con su aprendizaje, entrenamiento y práctica sistemática de sus habilidades (Conejeros et al., 2012; García-Cepero, et al., 2012; Llancavil y Lagos, 2016; Merino, Mathiesen, Mora, Castro y Navarro, 2014). 
12 RELACIÓN ENTRE AUTOESTIMA Y ESTRATEGIAS DE REGULACIÓN EMOCIONAL EN ESTUDIANTES CON ALTA CAPACIDAD QUE PARTICIPAN DE UN PROGRAMA DE ENRIQUECIMIENTO EXTRACURRICULAR CHILENO - G. Navarro Saldaña, G. Flores-Oyarzo y J. Rivera Illanes

Por tanto, es posible reconocer que los programas de enriquecimiento para estudiantes con alto potencial intelectual no solo consideran las necesidades educativas especiales asociadas a sus cualidades cognitivas, sino que, además, reconocen las necesidades socioafectivas de estos estudiantes.

\section{Características socioafectivas en la alta capacidad}

Ahora bien, aun cuando, en el contexto nacional, la generación de conocimiento en torno a las características socioafectivas de estudiantes con AC es escasa (Gould, Sánchez-Gómez y Breso, 2019), es posible describir ciertas cualidades socioafectivas características de estudiantes con alto potencial intelectual, entre ellas, una alta sensibilidad a las emociones y sensaciones de otros; intensidad emocional, caracterizada por la expresión de emociones de manera más intensa; intensidad sensorial, manifestada en elevado placer o displacer ante sabores, olores, sonidos particulares; sentimiento de orgullo por el logro exitoso de tareas desafiantes, así como también experimentar altos niveles de ansiedad al enfrentar un problema académico difícil, pudiendo estar acompañado de culpa o vergüenza cuando no logra cumplir con las expectativas de los demás; idealismo; desarrollo temprano del sentido de justicia y la moralidad, y además preocupación temprana por la igualdad entre personas y las causas sociales justas. Por otro lado, pueden ser perfeccionistas y autoexigentes, basados en un alto sentido crítico de sí mismos (Albes et al., 2013; Barrera, Durán, González, y Reina, 2008; Campo, 2016; Clark \& Callow, 2002; Mendioroz et al., 2019; Pirovano, 2019; Sánchez, 2014; Valadez y Ávalos, 2010; Zeidner \& Matthews, 2017).

Además, se ha planteado que estudiantes con AC, al visualizarse como personas distintas de sus pares, pueden desarrollar problemas de ansiedad e inseguridad, sintiéndose solos y aislados, pudiendo así ser más vulnerables a desarrollar problemas socioafectivos (Gould et al., 2019; Matthews, Lin, Zeidner \& Roberts, 2018).

En un estudio realizado en Chile por González, Gómez-Arízaga y Conejeros-Solar (2017), en el que caracterizan el perfeccionismo en estudiantes con AC, se evidencia que aquellos estudiantes que cuentan 
con un perfeccionismo desadaptativo consideran el entorno como un espacio en el que deben demostrar múltiples potencialidades, donde sus capacidades son configuradas como herramientas para obtener beneficios en torno a movilidad económica y social, junto con el reconocimiento social. A ello se suma la señalización de un sistema educativo que no siempre atiende sus necesidades, con expectativas docentes estereotipadas sobre ellos. Estudiantes con perfeccionismo desadaptativo pueden ser sumisos a las condiciones que impone el contexto social, llevándolos a sucumbir ante la presión externa, sacrificando sus predilecciones personales. Mientras que estudiantes con AC que poseen perfeccionismo adaptativo muestran una visión más próspera del entorno, visualizando a la sociedad como un espacio de crecimiento; refieren altos niveles de autonomía en diversos niveles y un notable desarrollo moral.

Por otro lado, en un estudio realizado por Gómez-Arízaga et al. (2020) sobre dimensiones socioemocionales en estudiantes chilenos con AC y en otro realizado por Roa-Tampe et al. (2020), en el que se estudió la influencia de los estereotipos de género y la participación en programas de enriquecimiento sobre el autoconcepto en estudiantes con AC, se encontró que las mujeres puntúan más bajo que los hombres en autoconcepto global y académico, y que los estudiantes que participan de programas de enriquecimiento puntúan más bajo en autoconcepto global en comparación con a aquellos que no lo hacen. Esto último podría ser explicado por la tesis presentada en Marsh et al. (2018), denominada "Big Fish Little Pond Effect", la cual plantea que, debido a un efecto de comparación con pares, los estudiantes con AC que participan de ambientes desafiantes académicamente, como son los programas de enriquecimiento, tienen menor autoconcepto académico que el que presentarían en un ambiente menos desafiante.

\section{Autoconcepto y autoestima}

Siguiendo esta línea y pasando a la revisión teórica de las variables a medir en estudiantes con AC, se encuentran el autoconcepto y la autoestima. Si bien es posible encontrar en la literatura el uso de estos constructos de manera intercambiable o indistinta, diversos autores 
14 RELACIÓN ENTRE AUTOESTIMA Y ESTRATEGIAS DE REGULACIÓN EMOCIONAL EN ESTUDIANTES CON ALTA CAPACIDAD QUE PARTICIPAN DE UN PROGRAMA DE ENRIQUECIMIENTO EXTRACURRICULAR CHILENO - G. Navarro Saldaña, G. Flores-Oyarzo y J. Rivera Illanes

ofrecen una conceptualización clara y diferenciada de cada uno de ellos (Roa, 2013; Clark \& Dunbar, 2003). Al ser la autoestima global nuestra variable de interés, profundizaremos en su conceptualización y diferenciación teórica respecto del autoconcepto.

La autoestima puede entenderse como aquella actitud y evaluación interna que hacen las personas de sí mismas, relacionada con el sentido de valía personal y la forma positiva o negativa en que se perciben dentro del mundo, sustentada en los pensamientos, sentimientos y experiencias vitales (Marmolejo y Romero, 2016; Mejía, 2007; Montoya y Sol, 2001; Vicent, 2018).

Al igual que el autoconcepto, es multidimensional y jerárquica, de manera que las personas se autovaloran en mayor o menor medida respecto de las diferentes áreas o dimensiones en que se desenvuelven, conformando así su autoestima global sobre la base de una autoestima física, social, académica, etc., y cada una ellas aportará en distinto nivel a la autoestima global según su nivel de jerarquía (Massenzana, 2017; Vicent, 2018).

A su vez, la autoestima global estaría conformada por tres componentes centrales: el componente cognitivo, que alude a las percepciones e ideas sobre sí mismo, y puede ser entendido como el autoconcepto, el cual cumple un rol en el origen y desarrollo de la autoestima; el componente afectivo, relacionado con la valoración positiva o negativa del sí sismo, y finalmente el componente conductual, relativo a comportarse coherente y consecuentemente consigo mismo, relacionado con la autoafirmación y la búsqueda de reconocimiento de los demás (Lorenzo, 2007).

De manera que el autoconcepto se entiende como un componente que forma parte de la autoestima, siendo parte del aspecto cognitivo de esta última, en tanto actúa como recolector de información, percepciones e ideas respecto del sí mismo.

Por otro lado, habría un factor social influyente en el desarrollo de la autoestima global, en tanto surge a partir de la interacción con otros en su construcción dinámica de integración de variables 
contextuales e internas (Enrique y Muñoz, 2014; Morales y González, 2014; Gómez, 2012; Naranjo, 2007; Acosta, 2004; Ramos, 2004).

Con base en el análisis e integración de las propuestas conceptuales expuestas, en este estudio se entenderá la autoestima global como un constructo multidimensional y jerárquico, que refiere a la autoevaluación y valoración, ya sea positiva o negativa, que hace una persona sobre sus cualidades, habilidades y características percibidas. Esta valoración no solo surge a partir de variables internas, como puede ser la percepción de las cualidades personales, sino también de variables contextuales y sociales, en tanto la persona se encuentra en un entorno social. Está compuesta por dimensiones según las áreas de actividad y desarrollo humano, pudiendo surgir así la autoestima académica, social, personal, familiar, laboral, etc., y tiene un componente cognitivo (autoconcepto), uno afectivo y uno conductual.

Respecto del impacto de la autoestima en los estudiantes, se ha propuesto que alumnos con alta autoestima suelen tener una buena capacidad para enfrentar problemas y dificultades, actúan acorde a sus creencias, valores y principios, son abiertos al aprendizaje y a nuevas experiencias, son seguros de sí mismos y de sus capacidades, y han construido un concepto realista tanto de sus fortalezas como de sus debilidades. Por otro lado, los estudiantes con baja autoestima suelen ser inseguros a la hora de tomar decisiones, se sienten insatisfechos con lo que hacen e incapaces de hacer cosas por sí mismos, y no creen en sus habilidades para enfrentar problemas o desafíos nuevos, tendiendo a ser más cerrados al diálogo y a reconocer sus errores (Cobos, 2011; Sánchez y Solís, 2010).

La autoestima en niños, niñas y jóvenes es un proceso dinámico, influenciado por el entorno, las interacciones sociales, las personas que les rodean y el contexto en general, siendo estas experiencias relacionales de gran relevancia para el fortalecimiento de la autoestima y de la construcción de vínculos con los otros (Rodríguez-Garcés, Gallegos y Padilla, 2021). 
16 RELACIÓN ENTRE AUTOESTIMA Y ESTRATEGIAS DE REGULACIÓN EMOCIONAL EN ESTUDIANTES CON ALTA CAPACIDAD QUE PARTICIPAN DE UN PROGRAMA DE ENRIQUECIMIENTO EXTRACURRICULAR CHILENO - G. Navarro Saldaña, G. Flores-Oyarzo y J. Rivera Illanes

En este sentido, la autoestima se iría complejizando a medida que los niños crecen y se ven influenciados por otros factores, como el rendimiento académico, la opinión de los pares, las expectativas que los adultos o figuras significativas tienen sobre ellos, la apariencia física y las normas sociales, dándose la tendencia a una disminución de la autoestima global (Lagos y Palma, 2018). En un estudio realizado por Marchant, Milicic y Pino (2017) se informa que la disminución de la autoestima hacia fines de la enseñanza básica se debe al incremento en la toma de conciencia de las propias limitaciones y al condicionamiento del entorno respecto de sus opciones futuras. Dicho estudio evidencia que estudiantes chilenos de quinto año básico tendrían una autoestima global de nivel medio, dando cuenta de que, a medida que se incrementa el nivel educativo en el análisis, vale decir, a mayor edad de los estudiantes, el nivel de autoestima global va en disminución.

En cuanto a la autoestima y su relación con el potencial intelectual, los estudios son escasos y contradictorios. Topçu \& Leana-Taşcilar (2018) presentan diversos estudios que informan de algunos hallazgos en esta temática, y en algunos de ellos se afirma que el potencial intelectual afectaría positivamente la autoestima, demostrando que estudiantes con AC gozarían de mejor autoestima que sus pares sin AC; otros afirman que esta autoestima más elevada sería específica para el área académica, por lo tanto, estos estudiantes presentarían una autoestima académica elevada. En esta misma línea, algunos estudios proponen que la autoestima académica estaría más elevada en aquellos estudiantes con AC que participan de programas de educación especial, en comparación a aquellos con AC que no participan de dichos programas. Por su parte, Ogurlu \& Sevim (2017) presentan estudios en los que se afirma que la autoestima social de estudiantes con AC sería más baja que su autoestima académica.

Estas contribuciones reportan que, si bien en el contexto internacional la literatura abunda respecto de la autoestima global de estudiantes con AC, en el ámbito latinoamericano hay escasez de conocimiento actualizado y generado sobre este tema. 
Sumado a lo anterior, no solo sería relevante estudiar el nivel de autoestima en estudiantes con AC, sino también su relación con la regulación emocional, pues en Rodríguez-Garcés et al. (2021) se propone que altos niveles de autoestima están asociados no solo a mayores niveles de satisfacción con la vida, sino que también contribuye a la regulación del comportamiento propio y hacia los demás. Por ello, se considera la regulación emocional, y específicamente las estrategias de regulación emocional, como una variable de interés en este estudio.

\section{Estrategias de regulación emocional}

En los últimos años ha habido un gran interés en la regulación emocional, pues está asociada a variables como el bienestar, el desarrollo psicológico y el desempeño académico (Gómez y Calleja, 2016).

Al ser las estrategias de regulación emocional la variable de interés de este estudio, se profundizará en ello por sobre la regulación emocional en sí misma; sin embargo, parece relevante presentar un breve marco conceptual con el cual se comprenderá esta última.

La regulación emocional (RE) puede entenderse como un conjunto de procesos, conscientes o inconscientes, automáticos o controlados, que permiten: monitorear, evaluar y modular las emociones, afectando su latencia, momento de aparición, magnitud, duración y sus respuestas conductuales, experienciales o fisiológicas; modificar el comportamiento y conductas para alcanzar metas; promover el bienestar individual y/o social y adaptarse al contexto, permitiendo disminuir, aumentar o mantener emociones tanto positivas como negativas, jugando un rol fundamental en el funcionamiento psicológico y en la salud mental de las personas (Bayo, 2016; Bisquerra, 2003; Del Valle, Betegón e Irurtia, 2019; Gómez y Calleja, 2016; Navarro, Vara, Cebolla y Baños, 2018).

Se entiende como un logro del desarrollo, pues requiere de cierta experiencia vital y la vivencia de un proceso progresivo y paulatino de interiorización de estrategias regulatorias que permitan 
18 RELACIÓN ENTRE AUTOESTIMA Y ESTRATEGIAS DE REGULACIÓN EMOCIONAL EN ESTUDIANTES CON ALTA CAPACIDAD QUE PARTICIPAN DE UN PROGRAMA DE ENRIQUECIMIENTO EXTRACURRICULAR CHILENO - G. Navarro Saldaña, G. Flores-Oyarzo y J. Rivera Illanes

al individuo tomar decisiones sobre sus propias emociones y las emociones de los demás, ocurriendo una transición desde una regulación externa hacia una de regulación interna, más compleja y caracterizada por una mayor autonomía e independencia, en la cual la persona interioriza y asume mecanismos de control (Bayo, 2016; Gómez y Calleja, 2016).

Sobre la base de las propuestas conceptuales expuestas, para fines de este estudio se comprenderá la "regulación emocional" como aquella serie de procesos automáticos o controlados, conscientes o inconscientes, que permiten a las personas monitorear y modular la latencia, duración, aparición y expresión de sus emociones, regular sus comportamientos para el logro de metas y su adaptación al contexto, disminuyendo, aumentando o manteniendo emociones positivas o negativas, y promoviendo así el bienestar individual y/o social.

Por su parte, por "estrategias de regulación emocional" se puede entender aquellos mecanismos de influencia por medio de los que se modifican o modulan las emociones, las cuales tienen un rol adaptativo en función de cada persona, sus propósitos y su contexto (Gómez y Calleja, 2016).

Existen diferencias individuales en la capacidad y forma de regular las emociones, siendo algunas más exitosas que otras (Pineda, Valiente, Chorot, Piqueras y Sandín, 2018). Las estrategias de regulación emocional que mayor apoyo empírico han recibido son la reevaluación cognitiva y la supresión expresiva (Navarro et al., 2018).

La reevaluación cognitiva $(\mathrm{RC})$ es una estrategia centrada en los antecedentes de la emoción y tiene como fin neutralizar el impacto emocional negativo o, bien, amplificar el estado emocional positivo; para ello se realiza la construcción de un nuevo significado de la situación elicitadora (Navarro et al., 2018; Pineda et al., 2018).

La supresión expresiva (SE), por su parte, es una estrategia centrada en la respuesta, e implica la modificación de la respuesta 
conductual de la persona frente a la emoción, sin reducir la experiencia emocional y manteniéndola presente. Refiere a la capacidad de la persona para inhibir el curso expresivo de la emoción (Gross \& John, 2003; Navarro et al., 2018; Pineda et al., 2018).

Tanto la RC como la SE impactan fuertemente en el funcionamiento psicológico de las personas (Navarro et al., 2018). El uso regular de estrategias de RC se relaciona con mejor funcionamiento interpersonal y mayor bienestar en general (Gross \& John, 2003), mientras que la SE con mayor número de síntomas depresivos, menos éxito en la recuperación del estado de ánimo y menos relaciones interpersonales (Navarro et al., 2018).

La manera en que las personas regulan sus emociones afecta su bienestar, su experiencia del estrés y sus relaciones (Pineda et al., 2018). Investigaciones han demostrado que aquellos estudiantes que presentan una alta emocionalidad negativa y no disponen de las habilidades regulatorias necesarias para modularla, tienen mayor probabilidad de expresar niveles altos de agresividad y dañar sus relaciones sociales, siendo la regulación emocional un determinante para una competencia social óptima (Navarro et al., 2018).

En cuanto a las estrategias de regulación emocional utilizadas por estudiantes con AC, éstas dependen de factores tales como la edad, el temperamento y características moduladas por medio de la experiencia, y marcada por los padres y el contexto. No obstante, las investigaciones sobre las estrategias que utilizan son escasas, encontrándose estudios en los que se propone que estudiantes con AC adquieren habilidades regulatorias a edades tempranas en comparación con otros, al utilizar su resiliencia e inteligencia en optar por estrategias de afrontamiento más efectivas para enfrentar el estrés (Sternberg, 1985; Campo, 2016). Y otras en las que se plantea que estos estudiantes son capaces de poner en marcha estrategias de reevaluación cognitiva de forma espontánea, aun cuando éstas hayan sido asociadas con estrategias utilizadas principalmente por adultos frente a las experiencias emocionales, lo cual les permitiría desarrollar comportamientos eficaces de solución de problemas y 
20 RELACIÓN ENTRE AUTOESTIMA Y ESTRATEGIAS DE REGULACIÓN EMOCIONAL EN ESTUDIANTES CON ALTA CAPACIDAD QUE PARTICIPAN DE UN PROGRAMA DE ENRIQUECIMIENTO EXTRACURRICULAR CHILENO - G. Navarro Saldaña, G. Flores-Oyarzo y J. Rivera Illanes

reinterpretaciones adaptativas de situaciones estresantes, todo ello resultando en un mejor manejo del estrés (Sowa et al., 1994).

Dadas las características socioafectivas de los estudiantes con AC y el impacto que tiene la autoestima y la regulación emocional en el bienestar y la salud mental de las personas, parece relevante estudiar ambas variables en estudiantes que cuenten con AC, permitiendo generar conocimiento científico que aporte a la construcción de estrategias y oportunidades educativas que contribuyan a la satisfacción de necesidades educativas especiales de estos estudiantes, y ayudarles a transformar su potencial intelectual en talento académico.

En esa línea, este estudio buscó comprender la relación entre el nivel de autoestima global y el uso de estrategias de regulación emocional en estudiantes con alta capacidad que participan de un Programa de Enriquecimiento Extracurricular de la Universidad de Concepción (Talentos UdeC), intentando responder a las siguientes preguntas de investigación: ¿Cómo es la autoestima global de estudiantes con alta capacidad que participan de un programa de enriquecimiento extracurricular? ¿Qué tipo de estrategias de regulación emocional utilizan? ¿Cómo es la relación entre la autoestima global percibida y las estrategias de regulación emocional utilizadas por estos estudiantes?

\section{Metodología}

\subsection{Diseño de investigación}

Estudio de carácter descriptivo-correlacional de naturaleza cuantitativa, dado que su propósito fue comprender la relación entre el nivel de autoestima y el tipo de estrategia de regulación emocional utilizada en estudiantes con alta capacidad, siendo apropiado entonces un estudio que caracterice los fenómenos, para luego analizar su relación y encontrar posibles explicaciones de dichas interacciones. El estudio es cuantitativo, en tanto los datos fueron recogidos por instrumentos de medición y analizados con métodos estadísticos descriptivos y correlacionales. 
Sobre la base de los supuestos que emergen a partir de la revisión teórica expuesta, nacieron las siguientes hipótesis:

- El nivel de autoestima global de estudiantes con alta capacidad, participantes de un programa de enriquecimiento extracurricular, será igual o mayor a la población estandarizada chilena.

- Estudiantes con alta capacidad, participantes de un programa de enriquecimiento extracurricular, utilizan estrategias de reevaluación cognitiva por sobre estrategias de supresión expresiva.

- A mayor autoestima, mayor será el uso de estrategias de reevaluación cognitiva como mecanismo de regulación emocional.

\subsection{Muestra y población}

Se realizó un muestreo intencionado por conveniencia, no probabilístico. Los participantes fueron escogidos basándose en la accesibilidad de la muestra y en los siguientes criterios de inclusión: ser participantes de primer ciclo del programa de enriquecimiento extracurricular; cursar entre quinto y sexto año básico; tener entre 10 y 12 años; cursar estudios en establecimientos públicos, particulares subvencionados o particulares pagados, y provenir de al menos tres comunas diferentes de la región del Biobío.

La muestra estuvo constituida por 37 participantes entre 10 y 12 años, de los cuales 21 eran mujeres y 16 hombres, todos identificados con alta capacidad y participantes de un programa de enriquecimiento extracurricular (Talentos UdeC).

Según Argibay (2009), que una muestra no sea probabilística no afectaría la validez externa de un estudio, pues ésta posee gran validez ecológica en la medida en que se tomen precauciones respecto de sus características en función de la población, y se determine claramente a quienes se podría generalizar los resultados del estudio. Plantea, además, que una muestra de tamaño importante es deseable, más no excluyente para la calidad de la investigación.

Si bien la muestra fue pequeña para un estudio correlacional, ella estuvo conformada por estudiantes que cursan estudios en 
22 RELACIÓN ENTRE AUTOESTIMA Y ESTRATEGIAS DE REGULACIÓN EMOCIONAL EN ESTUDIANTES CON ALTA CAPACIDAD QUE PARTICIPAN DE UN PROGRAMA DE ENRIQUECIMIENTO EXTRACURRICULAR CHILENO - G. Navarro Saldaña, G. Flores-Oyarzo y J. Rivera Illanes

distintos tipos de establecimiento educativos según el tipo de financiamiento que reciben —establecimientos públicos, particulares subvencionados y particulares pagados-, provenían de diversas comunas de la región del Biobío, todos contaban con alta capacidad y eran participantes de un programa de enriquecimiento extracurricular, siendo estos últimos dos criterios de gran relevancia, pues el estudio se centró en medir las variables específicamente en estudiantes con alta capacidad y que fueran parte de dichos programas.

\subsection{Instrumentos}

7.3.1. Escala de Autoconcepto para niños y jóvenes de Piers-Harris, en la versión de Gorostegui

La Escala de Autoconcepto para niños y jóvenes de Piers-Harris ha sido uno de los instrumentos más difundidos y utilizados en diversos contextos para medir la autoestima en niños y explorar su relación con otras variables (Cardenal \& Fierro, 2003; Moreno et al., 2011). Para medir la autoestima global, en este estudio se utilizó la versión adaptada y estandarizada en Chile por María Elena Gorostegui (1992, citado en Gorostegui y Dörr, 2004), que consta de 70 ítems formulados y adecuados en complejidad para niños en edad escolar entre tercero y sexto básico, los cuales se deben contestar mediante respuestas Sí o NO.

Los ítems de la escala fueron puntuados positiva o negativamente reflejando la dimensión autoevaluativa, de manera que un alto puntaje en la escala total sugiere una autovaloración positiva, mientras un bajo puntaje una autoevaluación negativa (Gorostegui y Dörr, 2004).

Con base en los descriptores propuestos por Piers (1984, citado en Gorostegui, 2004) y utilizado por Gorostegui (2004), es posible clasificar desde "Muy bajo" a "Alto".

En cuanto a su confiabilidad, Gorostegui y Dörr (2004) informaron que la escala total tiene un coeficiente de ,855 puntos en el Coeficiente de Confiabilidad KR20, con un error estándar de 
medida de 3,48 puntos, dando cuenta de adecuadas propiedades psicométricas como instrumento de medición.

\subsubsection{Cuestionario de Regulación Emocional para niños y} adolescentes (ERQ-CA), versión revisada por Gullone y Taffe (2012)

Para medir las estrategias de regulación emocional se utilizó el Cuestionario de Regulación Emocional para niños y adolescentes (ERQ-CA), que comprende diez ítems y evalúa dos tipos de estrategias de regulación emocional: reevaluación cognitiva y supresión emocional.

Sus ítems se agrupan en dos factores, seis refieren a reevaluación cognitiva y los cuatro restantes a supresión expresiva (Pineda et al., 2018; Navarro et al., 2018). En ellos, la persona expresa su nivel de acuerdo o desacuerdo respecto de la forma en que suele regular sus emociones, respondiendo en una escala tipo Likert de siete puntos, siendo 1 completamente en desacuerdo y 7 completamente de acuerdo (Pineda et al., 2018).

Para su análisis se puntuaron y sumaron puntajes de cada una de las escalas por separado (reevaluación cognitiva y supresión expresiva), interpretando de manera independiente el puntaje obtenido en cada una de ellas. A mayor puntaje, mayor uso de esa estrategia de regulación emocional frente a estímulos emocionales.

En este estudio se utilizó una versión revisada del instrumento original por Gullone y Taffe (2012), optimizada en su redacción y longitud para una población infantil, siendo traducida al español por el equipo investigador. Las revisiones de los autores incluyeron la simplificación de la redacción del ítem y la reducción en la longitud de la escala de respuesta de siete a cinco puntos, siendo 1 muy en desacuerdo y 5 totalmente de acuerdo.

Las puntuaciones más altas en cada escala indican un mayor uso de la estrategia de regulación emocional correspondiente.

En cuanto a sus cualidades psicométricas, en un estudio realizado por Gullone y Taffe (2012), para el factor reevaluación 
24 RELACIÓN ENTRE AUTOESTIMA Y ESTRATEGIAS DE REGULACIÓN EMOCIONAL EN ESTUDIANTES CON ALTA CAPACIDAD QUE PARTICIPAN DE UN PROGRAMA DE ENRIQUECIMIENTO EXTRACURRICULAR CHILENO - G. Navarro Saldaña, G. Flores-Oyarzo y J. Rivera Illanes

cognitiva se obtuvo un Alfa de Cronbach de, 83 puntos, y para el factor supresión expresiva se observó uno de, 75 puntos. Los autores concluyeron que el instrumento cuenta con adecuada consistencia interna, validez de constructo y validez convergente, constituyéndose en un instrumento de medición con adecuadas propiedades psicométricas.

\subsection{Procedimiento}

Para acceder a la muestra se solicitó autorización a la Dirección del programa de enriquecimiento, quienes accedieron a la realización del estudio y con quienes se comprometió una intervención psicoeducativa en función de la información recolectada y los resultados obtenidos. El consentimiento fue firmado por los padres al aceptar los términos de participación de sus hijos e hijas en el programa de enriquecimiento extracurricular.

Se informó a los estudiantes la intención de que fueran partícipes de un estudio en el que se recogería información sobre algunas de sus cualidades socioafectivas, entre ellas su autoestima y sus estrategias para regular emociones, explicándoles que su participación era voluntaria e indicándoles la fecha, horario y el tipo de participación (responder a dos cuestionarios).

Llegada la fecha de aplicación de instrumentos, los estudiantes que accedieron a participar de manera voluntaria concedieron su asentimiento informado, que fue leído con ellos de manera conjunta, fue explicado y se respondieron preguntas. El asentimiento dio cuenta de la voluntariedad de la participación, resguardo de la confidencialidad y uso de la información recogida con fines investigativos. La confidencialidad de la información fue resguardada por la investigadora responsable de este estudio.

La aplicación de los instrumentos se realizó en una sola jornada, con una duración de una hora y quince minutos, aproximadamente, en un espacio físico y horario asignado dentro de la jornada de participación en el programa de enriquecimiento, sin afectar la ejecución normal de las clases e instancias formativas disciplinares. 
Los cuestionarios fueron respondidos de manera anónima, vale decir, los estudiantes no debían escribir su nombre en el cuestionario, de esa manera se pretendía disminuir el factor de deseabilidad social en las respuestas dadas por los estudiantes.

Dado que los cuestionarios fueron respondidos de manera anónima, pero se requería de cierta identificación para correlacionar los datos recogidos de ambos instrumentos, se implementó un sistema de rotulación. Con este sistema se buscó que los datos de ambos instrumentos correlacionados correspondieran efectivamente a las respuestas del mismo estudiante, es decir, que el estudiante que respondía al cuestionario rotulado con el número "200" en la Escala de Autoconcepto de Piers-Harris, también respondía el cuestionario rotulado con el número "200" en el Cuestionario de Regulación Emocional.

El primer instrumento entregado fue la Escala de Autoconcepto de Piers-Harris. Una vez el estudiante finalizó este cuestionario, se le hizo entrega del Cuestionario de Regulación Emocional rotulado con el mismo número que le fue asignado en la Escala de Autoconcepto.

Finalizada la aplicación, se agradeció la participación a los estudiantes, los datos fueron ingresados a la base de datos, analizados y, sobre la base de los resultados obtenidos, se ofreció un taller de intervención psicoeducativa voluntario.

\subsection{Análisis de datos}

Sobre el análisis de los datos se realizó el análisis descriptivo, con el fin de identificar el nivel de autoconcepto global y la estrategia de regulación emocional utilizada por los participantes. Posteriormente se hizo análisis correlacional a través del coeficiente de Pearson, con el objetivo de reconocer la relación entre ambas variables. Para ello se utilizó el software JASP. 
26 RELACIÓN ENTRE AUTOESTIMA Y ESTRATEGIAS DE REGULACIÓN EMOCIONAL EN ESTUDIANTES CON ALTA CAPACIDAD QUE PARTICIPAN DE UN PROGRAMA DE ENRIQUECIMIENTO EXTRACURRICULAR CHILENO - G. Navarro Saldaña, G. Flores-Oyarzo y J. Rivera Illanes

\section{Resultados}

\subsection{Análisis descriptivo de la Escala de Autoconcepto de Piers-Harris}

Del total de participantes, un 84\% (31 estudiantes) presentaron un puntaje total superior a lo esperado en la mediana de la población estandarizada de escolares, encontrándose sobre el percentil 50 de acuerdo con los baremos establecidos por Gorostegui (2004).

Esto daría cuenta de que estudiantes con alta capacidad, participantes de un programa de enriquecimiento extracurricular, poseen un elevado nivel de autoestima en relación con la mediana establecida por Gorostegui dentro del contexto chileno.

\subsection{Análisis descriptivo del Cuestionario de Regulación Emocional ERQ-CA}

Los análisis realizados dan cuenta de que la estrategia de reevaluación cognitiva puntúa en promedio 25,05 puntos, mientras que la de supresión expresiva puntúa 11,94 puntos, demostrando así una tendencia a la utilización de estrategias de reevaluación cognitiva por sobre las de supresión expresiva. Mediante la prueba T Student, se comprueba que la diferencia de medias entre ambas estrategias de regulación emocional es estadísticamente significativa, con un valor p de ,001 (tabla 1).

Tabla 1

Prueba T para confirmar significancia estadística de medias

\begin{tabular}{cccccc}
\hline Medición 1 & Medición 2 & t & df & p \\
\hline RC & ES & 16.261 & 36 & $<, 001$ \\
\hline
\end{tabular}

Fuente: Creación propia.

\subsection{Análisis correlacional de ambas variables}

Al analizar la correlación entre las variables por medio del coeficiente de correlación de Pearson, no se encuentra correlación estadísticamente significativa entre la autoestima global y la estrategia de regulación emocional "reevaluación cognitiva". Sin embargo, 
existe una correlación negativa, estadísticamente significativa, entre la autoestima global y la supresión expresiva como estrategia de regulación emocional (valor p 0,$011 ; \alpha<0,05$ ), estableciéndose una relación inversa entre ambas, vale decir, a mayor valor en el nivel de autoestima global, menor valor en el uso de estrategias de regulación emocional enfocadas en la supresión de las emociones (tabla 2).

Tabla 2

Correlación negativa de Pearson entre puntaje Escala de Piers-Harris y estrategias de regulación emocional

\begin{tabular}{llccc}
\hline & Total & $\begin{array}{c}\text { Reevaluación } \\
\text { cognitiva }\end{array}$ & $\begin{array}{c}\text { Supresión } \\
\text { expresiva }\end{array}$ \\
\hline Total & Pearson`s r & - & & \\
Reevaluación & Valor de p & - & & \\
cognitiva & Pearson`s r & $-0,103$ & - & \\
Supresión & Pearson`s $\mathrm{p}$ & 0,272 & - & - \\
expresiva & Valor de p & $-0,374$ & 0,251 & - \\
\hline
\end{tabular}

Nota: Todas las pruebas son de una cola, para una correlación negativa.

Fuente: Creación de las autoras.

\section{Discusión}

Los resultados de este estudio permiten, de manera cautelosa, mostrar que estudiantes entre 10 y 12 años, con alta capacidad (AC) y que participan de un programa de enriquecimiento para estudiantes con alto potencial intelectual, cuentan con un nivel de autoestima global elevado y superior a la mediana estandarizada en población chilena, comprobando así la primera hipótesis de esta investigación: "El nivel de autoestima global de estudiantes con alta capacidad, participantes de un programa de enriquecimiento extracurricular, será igual o mayor a la población estandarizada chilena". Esto coincide con los planteamientos de los estudios presentados en Topçu \& LeanaTaşcilar (2018), en los cuales se evidencia que estudiantes con AC gozarían de mejor autoestima que sus pares.

Aun cuando en esta etapa del ciclo vital ocurren cambios profundos que afectan las relaciones interpersonales, la regulación emocional y la personalidad, y existe una gran influencia del entorno y las opiniones de los pares en la autoestima de los infantes (Delgado, 
28 RELACIÓN ENTRE AUTOESTIMA Y ESTRATEGIAS DE REGULACIÓN EMOCIONAL EN ESTUDIANTES CON ALTA CAPACIDAD QUE PARTICIPAN DE UN PROGRAMA DE ENRIQUECIMIENTO EXTRACURRICULAR CHILENO - G. Navarro Saldaña, G. Flores-Oyarzo y J. Rivera Illanes

2009; Di Blas \& Cepollaro, 2017; López, 2018; Mansilla, 2000; Papalia et al., 2012; Quispe, 2019; Sabatier et al., 2017), es posible hipotetizar que estos estudiantes aún gozan de una autoestima elevada debido a la edad en que se encuentran, pues, según los hallazgos de Marchant et al. (2017), en quinto año básico aún habría una autoestima media, y la profundización de los cambios y disminución de la autoestima estaría en séptimo y octavo básico.

Por otro lado, pese a que hay estudios que dan cuenta de que estudiantes con AC que participan de programas de enriquecimiento tienen un autoconcepto menor a aquellos que no participan de dichos estudios (Gómez-Arízaga et al., 2020; Roa-Tampe et al., 2020), el cual, al ser el componente cognitivo de la autoestima global, podría afectarla negativamente y se podría dar el efecto de "Big Fish Little Pond Effect" (Marsh et al., 2018), es posible hipotetizar que, dado que los estudiantes que participan de este estudio son alumnos de primer ciclo del programa de enriquecimiento, vale decir, estudiantes que en su mayoría cursan su primer año en el programa, no han sufrido los efectos del fenómeno descrito por Marsh et al. (2018), por lo que sería recomendable expandir este estudio incorporando a estudiantes de otros ciclos y que, por tanto, hayan cursado más años de estudio en el programa de enriquecimiento.

En cuanto las estrategias de regulación emocional, los resultados de este estudio informan que estos estudiantes implementan mayoritariamente estrategias de reevaluación cognitiva ( $\mathrm{RC}$ ) por sobre la supresión expresiva (SE), siendo estas diferencias estadísticamente significativas. Esto permite confirmar cautelosamente la segunda hipótesis propuesta en este estudio: "Estudiantes con alta capacidad, participantes de un programa de enriquecimiento extracurricular, utilizan estrategias de reevaluación cognitiva por sobre estrategias de supresión expresiva".

Estos hallazgos coinciden con los planteamientos de Sowa et al. (1994), quienes proponen que estudiantes con AC son capaces de poner en marcha estrategias de reevaluación cognitiva de manera espontánea, desarrollando comportamientos eficaces de solución de problemas y reinterpretaciones adaptativas de situaciones estresantes. 
Si bien esta estrategia es más probable en población adulta, porque requiere un proceso progresivo de interiorización de estrategias regulatorias que permite a las personas tomar decisiones sobre las propias emociones, transitando desde una regulación externa hacia una interna y más compleja (Bayo, 2016; Gómez y Calleja, 2016), los resultados de este estudio se relacionan con lo propuesto por Sternberg (1985) y Campo (2016), en cuanto a que los estudiantes con AC adquieren habilidades regulatorias a edades tempranas en comparación con sus pares.

El mayor uso de estrategias de RC por parte de estos estudiantes podría suponer, desde los planteamientos de Gross \& John (2003), que estos cuentan con un mejor funcionamiento interpersonal y son capaces de brindar un nuevo significado a la situación elicitadora de la emoción, amortiguando el impacto negativo de ésta o bien ampliando el estado emocional positivo (Navarro et al., 2018; Pineda et al., 2018).

La intensidad emocional, una característica socioafectiva común de estudiantes con AC (Albes et al., 2013; Barrera et al., 2008; Campo, 2016; Clark \& Callow, 2002; Mendioroz et al., 2019; Pirovano, 2019; Sánchez, 2014; Valadez y Ávalos, 2010; Zeidner $\&$ Matthews, 2017), puede estar vinculada con el menor uso de la estrategia de supresión expresiva, llevando a que estos estudiantes no logren inhibir el curso expresivo de la emoción, por lo cual no visualizarían dicha estrategia como una alternativa para regular sus emociones.

En cuanto a la relación entre autoestima y regulación emocional, los resultados de este estudio reportan una relación negativa y estadísticamente significativa entre la autoestima global y la estrategia supresión expresiva. Esto da cuenta de que no se cumple la tercera hipótesis planteada en este estudio: "A mayor autoestima, mayor será el uso de estrategias de reevaluación cognitiva como mecanismo de regulación emocional".

Los resultados podrían relacionarse con lo propuesto por Rodríguez-Garcés et al. (2021) respecto de que altos niveles 
30 RELACIÓN ENTRE AUTOESTIMA Y ESTRATEGIAS DE REGULACIÓN EMOCIONAL EN ESTUDIANTES CON ALTA CAPACIDAD QUE PARTICIPAN DE UN PROGRAMA DE ENRIQUECIMIENTO EXTRACURRICULAR CHILENO - G. Navarro Saldaña, G. Flores-Oyarzo y J. Rivera Illanes

de autoestima de los estudiantes aportan en la regulación del comportamiento propio y hacia los demás. En este sentido, es posible considerar que, a mayores niveles de autoestima, menor uso de la supresión expresiva como estrategia de regulación emocional, aportando así a menores índices de afectos negativos y depresión en los estudiantes. Del mismo modo, la supresión expresiva se relaciona con mayor número de síntomas depresivos, menos éxito en la recuperación del estado de ánimo y menos relaciones interpersonales (Navarro et al., 2018); sin embargo, estos estudiantes reportan elevados niveles de autoestima, lo cual implica una buena capacidad para enfrentar problemas y dificultades, seguridad en sí mismos y en sus capacidades, y la construcción de un concepto realista, tanto de sus fortalezas como de sus debilidades (Cobos, 2011; Sánchez y Solís, 2010), lo cual podría estar actuando como factor protector frente al uso de este tipo de estrategia.

Se reconoce que, aun cuando en Argibay (2009) se expresa que el tamaño de la muestra no es el único criterio para establecer la validez externa, y que la validez ecológica cobra gran valor en estudios con muestra no probabilística, en una muestra mayor, que incluya estudiantes con características distintas de los participantes de este estudio — como por ejemplo otro grupo etario—, se puede encontrar resultados distintos a los reportados en este estudio inicial. Por esta razón, se afirma que las primeras dos hipótesis de este estudio pueden ser confirmadas, pero de manera cautelosa.

Esto permite dar cuenta de algunas limitaciones en esta investigación, entre ellas, el tamaño de la muestra, el rango etario reducido de quienes participan de ésta y su baja experiencia como estudiantes del programa de enriquecimiento.

Entre las implicancias investigativas de este estudio, los resultados permiten dar luces iniciales e incipientes sobre el comportamiento de estas variables socioafectivas en estudiantes con AC, ofreciendo como futuras líneas de estudio de investigación el estudio del comportamiento de estas variables en estudiantes con AC que cursan estudios entre quinto año básico y cuarto año medio, es decir, entre 10 y 18 años de edad aproximadamente, que hayan 
cursado más años de estudio en el programa de enriquecimiento; comparar entre estudiantes con AC que participan y no participan de programas de enriquecimiento, con el fin de esclarecer el efecto de estos en las variables estudiadas, y comparar el comportamiento de las variables entre hombres y mujeres.

En términos prácticos, teniendo en consideración que, para transformar el potencial intelectual en talento académico se requiere de entornos estimulantes, que brinden oportunidades para su máximo y óptimo desarrollo (Acereda y Sastre, 1998; Mendioroz et al., 2019; Pérez et al., 2015; Rodríguez, 2013; Valadez y Ávalos, 2010; Worrell et al., 2019), que la manera en que las personas regulan sus emociones afecta su bienestar y sus relaciones (Pineda et al., 2018), y que altos niveles de autoestima están asociados a mayores niveles de satisfacción con la vida (Rodríguez-Garcés et al., 2021), este estudio busca aportar conocimiento respecto de las cualidades y necesidades de estudiantes con AC, con el propósito de favorecer la generación de espacios y contextos educativos donde éstos tengan la oportunidad formarse como personas integrales y con un elevado nivel de bienestar, viendo satisfechas no solo sus necesidades intelectuales y cognitivas, sino también sus necesidades socioafectivas.

\section{Conclusión}

Si bien en el mundo existe gran cantidad de investigaciones sobre autoestima y regulación emocional en estudiantes con AC, en el contexto chileno hay escasez de conocimiento acerca de esta temática que, por cierto, es de gran relevancia, no solo en términos de generación de conocimiento respecto de este alumnado en Chile, sino también por razones prácticos, con el fin de generar currículos y modelos educativos que permitan dar respuestas atingentes a las características y necesidades, tanto cognitivas e intelectuales como socioafectivas de estudiantes con AC.

Los elevados niveles de autoestima global reportados por los participantes de este estudio informan, de manera inicial e incipiente, que estudiantes con AC que aún se encuentran en etapa de infancia 
32 RELACIÓN ENTRE AUTOESTIMA Y ESTRATEGIAS DE REGULACIÓN EMOCIONAL EN ESTUDIANTES CON ALTA CAPACIDAD QUE PARTICIPAN DE UN PROGRAMA DE ENRIQUECIMIENTO EXTRACURRICULAR CHILENO - G. Navarro Saldaña, G. Flores-Oyarzo y J. Rivera Illanes

tardía no han visto afectada negativamente su autoestima a propósito de los cambios normativos esperados en su etapa evolutiva, pudiendo encontrarse estos efectos en edades más avanzadas dentro de esta misma etapa vital.

En cuanto a las estrategias de regulación emocional, en estos estudiantes la estrategia que sería mayoritariamente elegida sería la reevaluación cognitiva por sobre la supresión expresiva, lo cual podría explicarse desde las cualidades cognitivas propias de este estudiantado.

Finalmente, respecto de la relación entre ambas variables, los resultados de este estudio permiten comprender la relación entre el nivel de autoestima y la estrategia de regulación emocional utilizada por estos estudiantes, quienes, a mayor autoestima, usan menos la supresión expresiva como estrategia de regulación emocional.

Pese al pequeño tamaño de la muestra, este estudio es un aporte para la visibilización de las cualidades y necesidades socioafectivas de estudiantes con AC, y para relevar información que aporte a la implementación de modelos educativos que brinden oportunidades de aprendizaje, tanto cognitivo como socioafectivo.

Parece relevante, dada sus implicancias investigativas y prácticas, seguir ampliando y profundizando este tema de estudio en contexto chileno, incorporando una muestra mayor, con estudiantes de un rango etario más amplio, con más años de participación en programas de enriquecimiento, comparando el comportamiento de estas variables en estudiantes con AC que participan y no participan en un programa de enriquecimiento, y el comportamiento de ellas entre hombres y mujeres.

\section{Referencias}

Acereda, A. y Sastre, S. (1998). La superdotación. Ediciones Síntesis.

Acosta, R. y Hernández, J. (2004). La autoestima en la educación. Limite, 1(11), 82-95. Recuperado de: https://www.redalyc.org/pdf/836/83601104. pdf 
Albes, C., Aretxaga, L., Etxebarria, I., Galende, I., Santamaría, A., Uriarte, B. y Vigo, R. (2013). Orientaciones educativas. Alumnado con altas capacidades intelectuales. Departamento de Educación, Política Lingüistica y Cultura del país vasco. Recuperado de: https://r.issu. edu.do/l?l=10365W1W

Aribay, J. C. (2009). Muestra en Investigación Cuantitativa. Subjetividad y Procesos Cognitivos, 13(1), 13-29. Recuperado de: https://www.redalyc. org/pdf/3396/339630252001.pdf

Barrera, A., Durán, R., González, J. y Reina, C. L. (2008). Manual de atención al alumnado con necesidades especificas de apoyo educativo por presentar altas capacidades intelectuales. Sevilla: Junta de Andalucía. Recuperado de: https://redined.mecd.gob.es/xmlui/handle/11162/3171

Baslanti, U. \& McCoach, D. B. (2006). Gifted underachievers and factors affecting underachievement. Roeper Review, (28), 210-215. DOI: https://doi.org/10.1080/02783190609554366

Bayo, M. (2016). Estrategias cognitivas de regulación emocional, bienestar psicológico y sintomatología en adolescentes (Tesis de Grado, Universidad Nacional de Mar del Plata, Argentina). Recuperado de: http://200.0.183.210/handle/123456789/1148

Bisquerra, R. (2003). Educación emocional y competencias básicas para la vida. Revista de Investigación Educativa, 21(1), 7-43. Recuperado de: https://revistas.um.es/rie/article/view/99071/94661

Campo, M. (2016). Regulación emocional y habilidades sociales en niños con altas capacidades intelectuales (Memoria Doctorado, Universidad Complutense de Madrid, España). Recuperado de: https://eprints. ucm.es/38843/1/T37656.pdf

Cardenal, V. y Fierro, A. (2003). Componentes y correlatos del autoconcepto en la escala de Piers-Harris. Estudios de Psicología, 24(1), 101-111. DOI: https://doi.org/10.1174/021093903321329094

Céspedes, A. (2008). Educar las emociones. Educar para la vida. Ediciones B.

Clark, B. (2008). Growing up gifted: Developing the potential of children at home and at school. Merrill.

Clark, C. \& Callow, R. (2002). Educating the Gifted and Talented. David Futon Publishers.

Clark, P. \& Dunbar, S. (2003). Preliminary reliability and validity of a family care climate questionnaire for heart failure. Families, systems \& Health, 21(2), 281-291. DOI: https://doi.org/10.1037/1091-7527.21.3.281 
34 RELACIÓN ENTRE AUTOESTIMA Y ESTRATEGIAS DE REGULACIÓN EMOCIONAL EN ESTUDIANTES CON ALTA CAPACIDAD QUE PARTICIPAN DE UN PROGRAMA DE ENRIQUECIMIENTO EXTRACURRICULAR CHILENO - G. Navarro Saldaña, G. Flores-Oyarzo y J. Rivera Illanes

Cobos, F. (2011). Autoestima: características y manifestaciones en los estudiantes. Revista UNAVance, (1), 21-23. Recuperado de: https://r. issu.edu.do/1?l=10366iZU

Conejeros, M. L., Cáceres, P. y Riveros, A. (2012). Educación de Talentos Académicos en Chile: Una década de aprendizajes e investigación. En J. Catalán (Ed.), Investigación orientada al cambio en psicología educacional (pp. 39-74). Editorial Universidad de La Serena.

Di Blas, L. \& Cepollaro, A. (2017). Self-Esteem and Locus of Causality as Vulnerability Factors for the Development of Actual/Ideal SelfDiscrepancies in Late Childhood. Psychological Topics, 26(1), 241-259. Recuperado de: https://hrcak.srce.hr/index.php?show=clanak\&id_ clanak_jezik=266941

Del Valle, M., Betegón, E. e Irurtia, M. J. (2019). Efecto del uso de estrategias cognitivas de regulación emocional sobre la ansiedad en adolescentes españoles. Revista Suma Psicológica, 25(2), 153-161. DOI: http://dx.doi. org/10.14349/sumapsi.2018.v25.n2.7

Delgado, B. (2009). Psicología del Desarrollo. Volumen 2: Desde la infancia a la vejez. McGraw Hill.

Enrique, M. y Muñoz, R. (2014). El problema de la autoestima basado en la eficacia. Revista de Investigación en Psicología Social, 1(1), 52-58. Recuperado de: https://r.issu.edu.do/l?l=10367ZfW

García-Cepero, M. C., Proestakis, A. N., Lillo, A., Muñoz, E.M., López, C. y Guzmán, M. I. (2012). Caracterización de estudiantes desde sus potencialidades y talentos académicos en la región de Antofagasta, Chile. Universitas Psychologica, 11(4), 1327-1340. Recuperado de: https://revistas.javeriana.edu.co/index.php/revPsycho/article/ view/1658/3443

Gardner, H. (1993). Multiple intelligences. Basic Books.

Gagné, F. (2015). De los genes al talento: la perspectiva DMGT/CMTD. Revista de educación, (368), 12-39. DOI: http://dx.doi.org/10.4438/1988592X-RE-2015-368-289

Gómez, O. y Calleja, N. (2016). Regulación emocional: definición, red nomológica y medición. Revista mexicana de investigación en psicología, 8(1), 96-117. Recuperado de: https://r.issu.edu.do/l?l=10368SUg

Gómez-Arízaga, M. P., Navarro, M., Martin, A., Roa-Tampe, K., ConejerosSolar, M. L., Kronborg, L., Valdivia-Lefort, M., Castillo-Hermosilla, H. \& Rivera-Lino, B. (2020). Socio-emotional Dimensions in Gifted Chilean High School Students with Interests in STEM: Influence of Gender and University Enrichment Program Participation. EURASIA 
Journal of Mathematics, Science and Technology Education, 16(12). DOI: https://doi.org/10.29333/ejmste/9374

Gómez, O. I. y Calleja, B. N. (2017). Regulación emocional: Escalas de medición en español (revisión psicométrica). Revista Iberoamericana de Psicología, 10(2), 183-192. Recuperado de: https://revistas.ibero. edu.co/index.php/ripsicologia/article/view/1214

González, A., Gómez-Arízaga, M. P. y Conejeros-Solar, M.L. (2017). Caracterización del perfeccionismo en estudiantes con alta capacidad: un estudio de casos exploratorio. Revista de Psicología, 35(2). DOI: http://dx.doi.org/10.18800/psico.201702.008

Gorostegui, M. E. (2004). Género y autoconcepto: un análisis comparativo de las diferencias por sexo en una muestra de niño de E.G.B 1992-2003 (Tesis de maestría, Universidad de Chile). Recuperado de: https://r. issu.edu.do/l?1=10369kUv

Gorostegui, M. E. y Dörr, A. (2004). La escala de evaluación del autoconcepto para niños, de Piers Harris: actualización de normas. Castalia, revista de psicología de la Academia, (7), 81-97. Recuperado de: http:// bibliotecadigital.academia.cl/handle/123456789/2442

Gorostegui, M. E. y Dörr, A. (2005). Género y Autoconcepto: Un Análisis Comparativo de las Diferencias por Sexo en una Muestra de Niños de Educación General Básica (EGB) (1992-2003). Psykhe, 14(1), 151-163. DOI: http://dx.doi.org/10.4067/S0718-22282005000100012

Gould, A., Sánchez-Gómez, M. y Breso, E. (2019). Inteligencia Emocional y altas capacidades. Ágora de Salut, (6), 159-167. DOI: http://dx.doi. org/10.6035/agorasalut.2019.6.17

Gross, J. (1998). The emerging field of emotion regulation: an integrative review. Review of General Psychology, 2(3), 271-299. DOI: https://doi. org/10.1037/1089-2680.2.3.271

Gross, J. \& John, O. (2003). Individual differences in two emotion regulation processes: Implications for affect, relationships, and well-being. Journal of Personality and Social Psychology, 85(2), 348-362. DOI: https://doi. org/10.1037/0022-3514.85.2.348

Gullone, E. \& Taffe, J. (2012). The Emotion Regulation Questionnaire for Children and Adolescents (ERQ-CA): A psychometric evaluation. Psychological Assessment, 24(2), 409-417. DOI: https://doi.org/10.1037/ a0025777

Lagos, N. y Palma, M. (2018). Diferencias en Ansiedad Escolar, Autoestima y Perfeccionismo en función del nivel escolar y el sexo en estudiantes chilenos de educación primaria. Revista Reflexión e Investigación 
36 RELACIÓN ENTRE AUTOESTIMA Y ESTRATEGIAS DE REGULACIÓN EMOCIONAL EN ESTUDIANTES CON ALTA CAPACIDAD QUE PARTICIPAN DE UN PROGRAMA DE ENRIQUECIMIENTO EXTRACURRICULAR CHILENO - G. Navarro Saldaña, G. Flores-Oyarzo y J. Rivera Illanes

Educacional, 1(1), 57-69. Recuperado de: http://revistas.ubiobio.cl/ index.php/REINED/article/view/3405

Llancavil, D. R. y Lagos, L. F. (2016). Importancia de la Educación Inclusiva para el Trabajo con Niños con Talento Académico. Perspectiva Educacional, Formación de Profesores, 55(1), 168-183. DOI: http:// dx.doi.org/10.4151/07189729-Vol.55-Iss.1-Art.391

López, C. (2018). Comportamiento agresivo y variables psicoeducativas en la infancia tardía. (Tesis para optar al grado de Doctora en Investigación Educativa, Universidad de Alicante). Recuperado de: http://rua.ua.es/ dspace/handle/10045/99377

Lorenzo, M. (2007). Autoconcepto y autoestima, conocer su construcción. Cieza: Charla-coloquio.

Lovecky, D. V. (2009). Moral sensitivity in young gifted children. In D. Ambrose \& T. Cross (eds.), Morality, Ethics, and Gifted Minds. Springer.

Marchant, M., Milicic, N. y Pino, M.J. (2017). La Autoestima en Alumnos de $3^{\circ}$ a $8^{\circ}$ Básico. Una Mirada por Nivel de Escolaridad y Género. Revista Iberoamericana de Evaluación Educativa, 10(2), 111-125. DOI: https:// doi.org/10.15366/riee2017.10.2.006

Mansilla, M. E. (2000). Etapas del Desarrollo Humano. Revista de Investigación en Psicología, 3(2). Recuperado de: http://ateneo.unmsm.edu.pe/ handle/123456789/2035

Marmolejo, A. y Romero, A. (2016). IMC en estudiantes de primer grado de primaria y su relación con la autoestima y el autoconcepto. PsicoEducativa: reflexiones y propuestas, 2(3), 37-39. Recuperado de: https://r.issu.edu.do/l?l=10370gad

Martín, M.P. (2004). Niños inteligentes. Guía para desarrollar sus talentos y altas capacidades. Palabra.

Massenzana, F. (2017). Autoconcepto y Autoestima: ¿Sinónimos o constructos complementarios? PSOCIAL, 3(1), 39-52. Recuperado de: https://r. issu.edu.do/1?l=10371yok

Marsh, H. W., Pekrun, R., Murayama, K., Arens, A. K., Parker, P. D., Guo, J. \& Dicke, T. (2018). An integrated model of academic self-concept development: Academic self-concept, grades, test scores, and tracking over 6 years. Developmental Psychology, 54(2), 263-280. DOI: https:// doi.org/10.1037/dev0000393

Matthews, G., Lin, J., Zeidner, M. \& Roberts, R. (2018). Emotional Intelligence and giftedness. In S. Pfeiffer, E. Shaunessy-Dedrick \& M. Foley-Nicpon (eds.), APA Handbook of Gftedness and Talent. American Psychology Association. 
McCoach, D. B. \& Siegle, D. (2003). Factors that differentiate underachieving gifted students from high-achieving gifted students. Gifted Child Quarterly, 47(2), 144-154. DOI: https://doi. org/10.1177/001698620304700205

Mendioroz, A. M., Rivero, P. y Aguilera, E. (2019). Una propuesta de formación docente para responder a las altas capacidades en la escuela inclusiva. Profesorado: Revista de currículum y formación del profesorado, 23(1), 265-284. DOI: https://dx.doi.org/ 10.30827/profesorado.v23i1.9154.

Merino, J. M., Mathiesen, M. E, Mora, O., Castro, G. y Navarro, G. (2014). Efectos del Programa Talentos en el desarrollo cognitivo y socioemocional de sus alumnos. Estudios pedagógicos, XL(1), 197-214. Recuperado de: https://scielo.conicyt.cl/pdf/estped/v40n1/art12.pdf

Miguel, A. y Moya, A. (2011). Conceptos generales del alumno de altas capacidades. En J. C. Torrego (coord.), Alumnos con altas capacidades y aprendizaje cooperativo. Un modelo de respuesta educativa (pp. 13-33). Fundación Pryconsa; Fundación SM. Recuperado de: https://r.issu. edu.do/l?l=10372VAR

Montoya, M.A. y Sol, C.E. (2001). Autoestima: Estrategias para vivir mejor con PNL y Desarrollo Humano. Editorial PAX MÉXICO.

Morales, M. y González, A. (2014). Resiliencia-Autoestima-Bienestar psicológico y Capacidad intelectual de estudiantes de cuarto medio de buen rendimiento de liceos vulnerables. Estudios pedagógicos, 40(1), 215-228. DOI: http://dx.doi.org/10.4067/S071807052014000100013

Moreno, J., Ángel, A., Castañeda, B., Castelblanco, P., López, N. y Medina, A. (2011). Autoestima en un grupo de niños de 8 a 11 años de un colegio público de la ciudad de Bogotá. Psychología. Avances de la disciplina, 5(2), 155-162. https://www.redalyc.org/articulo.oa?id=297224105013

Mruk, C. \& Skelly, T. (2017). Is Self-Esteem Absolute, Relative, or Functional? Implications for Cross Cultural and Humanistic Psychology. The Humanistic Psychologist, 45(4), 313-332. DOI: https://doi.org/10.1037/ hum0000075

Naranjo, M. (2007). Autoestima: un factor relevante en la vida de la persona y tema esencial del proceso educativo. Actualidades Investigativas en Educación, 7(3), 1-27. Recuperado de: https://www.redalyc.org/ pdf/447/44770311.pdf

Navarro, J., Vara, M., Cebolla, A. y Baños, R. (2018). Validación psicométrica del cuestionario de regulación emocional (ERQ-CA) en población adolescente española. Revista de Psicología Clínica con Niños y 
38 RELACIÓN ENTRE AUTOESTIMA Y ESTRATEGIAS DE REGULACIÓN EMOCIONAL EN ESTUDIANTES CON ALTA CAPACIDAD QUE PARTICIPAN DE UN PROGRAMA DE ENRIQUECIMIENTO EXTRACURRICULAR CHILENO - G. Navarro Saldaña, G. Flores-Oyarzo y J. Rivera Illanes

Adolescentes, 5(1), 9-15. Recuperado de: https://dialnet.unirioja.es/ servlet/articulo? codigo $=6272820$

Ogurlu, U. \& Sevim, M. N. (2017). The Opinions of Gifted Students about Leadership Training. Journal of Ethnic and Cultural Studies, 4(2), 41-52. Recuperado de: http://www.ejecs.org/index.php/JECS/article/view/73/ pdf

Papalia, D. E., Feldman, R. D. y Martorell G. (2012). Desarrollo Humano. Mc Graw Hill.

Pérez, E., Furlan, L., Heredia, D. y Lescano, C. (2015). Estrategias, instrumentos y programas para la identificación y educación del talento. Anuario de Investigaciones de la Facultad de Psicología, 2(1), 96-113. Recuperado de: https://revistas.unc.edu.ar/index.php/aifp/ article/viewFile/12860/13087

Piers, E. V. \& Harris, D. B. (1969). Piers-Harris children's self-concept scale. Western Psychological Services.

Pineda, D., Valiente, R. M., Chorot, P., Piqueras, J.A. y Sandín, B. (2018). Invarianza factorial y temporal del Cuestionario de Regulación Emocional (ERQ). Revista de Psicopatología y Psicología Clínica, (23), 109120. DOI: http://dx.doi.org/10.5944/rppc.vol.23.num.2.2018.21823

Pirovano, M. B. (2019). La inclusión educativa de niños, niñas y jóvenes talentosos. Perspectivas: Revista científica de la Universidad de Belgrano, 2(1), 38-50. Recuperado de: https://revistas.ub.edu.ar/index.php/ Perspectivas/article/view/51/52

Quispe, W. P. (2019). Infancia Intermedia (Tesis para optar al Título Profesional de Licenciado en Educación, Universidad Nacional de Educación). Recuperado de: https://repositorio.une.edu.pe/handle/UNE/3717

Ramos, M. (2004). Valores y autoestima: Conociéndose a sí mismo en un mundo de otros. Revista Educación en Valores, 1(1), 1-14. Recuperado de: https://dialnet.unirioja.es/descarga/articulo/2222555.pdf

Roa, A. (2013) La educación emocional, el autoconcepto, la autoestima y su importancia en la infancia. EDETANIA, 44, 241-257. Recuperado de: https://dialnet.unirioja.es/servlet/articulo? codigo $=4596298$

Roa-Tampe, K. A., Castillo-Hermosilla, H. C., Valdivia-Lefort, M. C., Briseño, M. P., Gómez-Arizaga, M. P., Navarro, M., Martin, A., Rivera-Lillo, B. y Conejeros-Solar, M. L. (2020). Autoconcepto y alta capacidad: influencia de estereotipos de género y programas de enriquecimiento. Revista Espacios, 41(17), 14-26. Recuperado de: http://revistaespacios. com/a20v4ln17/a20v41n17p14.pdf 
Rodríguez, R. (2016). El contexto escolar y la vulnerabilidad del alumno con alto potencial intelectual: una experiencia mexicana. Revista Nacional e Internacional de Educación Inclusiva, 9(2), 225-244. Recuperado de: http://www.revistaeducacioninclusiva.es/index.php/REI/article/ view/62/57

Rodríguez-Garcés, C. R., Gallegos, M. y Padilla, G. (2021). Autoestima en Niños, Niñas y Adolescentes chilenos: análisis con árboles de clasificación. Reflexiones, 100(1), 1-18. Recuperado de: https://revistas. ucr.ac.cr/index.php/reflexiones/article/view/43342/44755

Sabatier, C., Restrepo, D., Moreno, M., Hoyos, O. \& Palacio, J. (2017). Emotion Regulation in Children and Adolescents: concepts, processes and influences. Psicología desde el Caribe, 34(1), 75-90. Recuperado de: https://www.redalyc.org/pdf/213/21356010006.pdf

Sastre-Riba, S. (2014). Intervención psicoeducativa en la alta capacidad: funcionamiento intelectual y enriquecimiento extracurricular. Revista de Neurología, 58(Supl. 1), 89-98. Recuperado de: https://r.issu.edu. do/l? $=10375 \mathrm{Hqq}$

Sánchez, M. I. (2014). Intervención en competencias socio-emocionales en niños/ as y adolescentes con Sobredotación Intelectual (Tesis de pregrado, Universidad de Jaén).

Sánchez, B. y Solís, J. (2010). Autoestima en niños. Ángeles Psicológicos. Recuperado de: https://r.issu.edu.do/l?l=10376Ftq

Silverman, L. K. (2012). Asynchronous development: A key to counseling the gifted child. In T. L. Cross \& J. R. Cross (Eds.), Handbook for counselors serving students with gifts and talents: Development, relationships, school issues, and counseling needs/interventions. Prufrock Press.

Snyder, K. \& Linnenbrink-Garcia, L. (2013). A developmental, personcentered approach to exploring multiple motivational pathways in gifted underachievement. Educational Psychologist, 48(4), 209-228. DOI: https://doi.org/10.1080/00461520.2013.835597

Sowa, C. J., McIntire, J., May, K. M. \& Bland, L. (1994). Social and emotional adjustment themes across gifted children. Roeper Review, 17(2), 95-98. DOI: https://doi.org/10.1080/02783199409553633

Sternberg, R. \& Kaufman, S. (2011). The Cambridge handbook of intelligence. Cambridge University Press.

Tamayo, A. (1981). EFA: Escala Fatorial de Autoconceito. Arquivo Brasileiro de Psicologia, (33), 87-102. Recuperado de: https://r.issu.edu.do/ $1 ? \mathrm{l}=10377 \mathrm{gPj}$ 
40 RELACIÓN ENTRE AUTOESTIMA Y ESTRATEGIAS DE REGULACIÓN EMOCIONAL EN ESTUDIANTES CON ALTA CAPACIDAD QUE PARTICIPAN DE UN PROGRAMA DE ENRIQUECIMIENTO EXTRACURRICULAR CHILENO - G. Navarro Saldaña, G. Flores-Oyarzo y J. Rivera Illanes

Topçu, S. \& Leana-Taşcilar. (2018). The role of motivation and selfesteem in the academic achievement of Turkish gifted students. Gifted Education International, 34(1), 3-18. DOI: https://doi. org/10.1177/0261429416646192

Valadez, M. y Ávalos, A. (2010). Atención educativa a estudiantes sobresalientes y talentosos en escuelas inclusivas. En G. Giraldo y C. Núñez (eds.), Inclusión y Talento. Equidad en una educación de calidad. Programa de Inclusión y Talento en el Aula (pp. 25-35). Ediciones Buinaima.

Vicent, N. (2018). Estudio del autoconcepto y rendimiento en un aula de $6^{\circ}$ de primaria (Tesis para optar al grado de Maestro en Educación Primaria, Universitat Jaume I). Recuperado de: http://repositori.uji.es/ xmlui/handle/10234/177910

Whitmore, J. R. (1980). Giftedness, conflict, and underachievement. MA: Allyn $\&$ Bacon.

Worrell, F. C., Subotnik, R. F., Olszewski-Kubilius, P. \& Dixson, D. D. (2019). Gifted Students. Annual Review of Psychology, (70), 551-576. DOI: https://doi.org/10.1146/annurev-psych-010418-102846

Zeidner, M. \& Matthews, G. (2017). Emotional intelligence in gifted students. Gifted education international, 33(2), 163-182. DOI: https://doi. org/10.1177/0261429417708879

Recibido: 16/04/2021

Aceptado: 31/08/2021 